\title{
Scalar perceptions of distance in a multiobject binocular display
}

\author{
DONALD H. MERSHON, SUELLEN GRANBERRY-HAGER, KEVIN BARTLETT, \\ and BURNEY DeCAMP \\ North Carolina State University, Raleigh, North Carolina 27650
}

\begin{abstract}
Even in the absence of the usual cues to egocentric distance, a single visual object tends to be seen at an "egocentric reference distance" as a combined result of a perceptual tendency and the residual oculomotor cues. It has previously been shown that two objects, separated in depth by relative cues, tend to be seen with the far object located near this reference distance and the near object displaced toward the observer. The present research demonstrates that a similar perceptual positioning of a depth configuration will also occur despite the presence of several intermediate objects in the interval between the near and far objects. The possibility that fixation patterns may be important to this phenomenon is discussed.
\end{abstract}

Scalar perceptions of egocentric distance are possible even under reduced viewing conditions in which the majority of cues to distance are absent. A single object seen under such conditions will tend to appear at an intermediate (2- to 3-m) distance. Gogel (1969) suggested that this phenomenon reflected the operation of a perceptual factor that he termed the specific distance tendency (SDT). Other influences upon the apparent distance of such an object include the oculomotor cues to distance, that is, the accommodation and convergence of the eyes. Since some state of these oculomotor cues exists in any visual situation, they always serve to modify the perceptual outcome expected from the SDT. Gogel (1972) called the combined result of the SDT and such unavoidable oculomotor cues the egocentric reference distance (ERD). He then used this concept to explain the occurrence of scalar perceptions under reduced viewing conditions.

Although an isolated object would be expected to appear at approximately the reference distance, the present research was directed to the problem of how a configuration of two or more objects separated in depth is perceptually positioned with respect to the ERD. Gogel (1972) investigated this question using two points of light that were separated in stereoscopic depth. Direct (absolute) cues to the egocentric distances from the observer were purposely minimized, although the depth interval between the points was clearly specified by the relative cue of binocular disparity. With this configuration, Gogel found that the far point appeared near the egocentric reference distance (as it would if seen by itself), but the near point appeared closer than it would if seen by itself. Thus the perceived distance

Reprints may be requested from Donald H. Mershon, Department of Psychology, North Carolina State University, Raleigh, North Carolina 27650. of the farther point was unaffected by presenting it in a configuration. The perceived position of the near point, on the other hand, was significantly displaced in depth (toward the observer) by the addition of a more remote object to the display.

Mershon and Lembo (1977) confirmed these results and extended their generality by examining a slightly different display, in which the nearer object consisted of a small vertical rectangle, rather than another point of light. Again, when the near object (the rectangle) was presented in configuration with a binocularly farther point of light, the near object was perceptually displaced toward the observer relative to its position when seen in isolation.

Mershon, Voncannon, and Windes (1976) considered the same problem in a monocularly viewed display. In their experiment, the monocular cues of relative size, relative accommodation, and motion parallax, as well as height in the field, produced the configuration of objects distributed in depth. As in the other studies, the far object remained at approximately the ERD and the near object was perceptually displaced toward the observer relative to its position when seen alone. Mershon et al. (1976) concluded that a depth interval need not be generated by stereoscopic cues and that viewing need not be binocular in order for this displacement phenomenon to occur.

The present experiment was designed to extend the investigation of this effect to a more complex configuration of objects. Specifically, would the nearer object(s) in a configuration still be displaced as much toward the observer if the space between the front and back of the display were filled with intermediate objects as would be the case for a configuration composed of only a single near and a single far object? Alternatively, might the more structured display be perceptually "anchored" so that its center was positioned at the ERD? Such a 
result would involve displacements of both near and far points from their positions when seen in isolation.

As in previous studies of this question, it is important to be certain that absolute cues to the actual distances of the objects have been minimized before considering the effect of presenting the configuration. If such cues are available, then a statistically significant difference in the perceived distances of the individual objects should occur when those objects are presented in isolation. Only if the distance reports for these independent presentations do not differ significantly is it reasonable to interpret the results of the simultaneous presentations as bearing on the question of how a depth configuration will be perceptually anchored with respect to the ERD.

\section{METHOD}

\section{Apparatus}

Four green and eight yellow-orange LEDs were arranged in two identical rows extending radially away from the observer on a tabletop within a darkened visual alley. Two green LEDs (the near points) were positioned at a distance from the observer of $404 \mathrm{~cm}$ and laterally separated by $30 \mathrm{~cm}(4.25 \mathrm{deg})$. A second pair of green LEDs (the far points) was located $734 \mathrm{~cm}$ away from the observer with a lateral separation of $14.0 \mathrm{~cm}$ (1.09 deg). The yellow-orange LEDs were also arranged as pairs in the interval between the near points and the far points, creating the impression of two straight rows of LEDs extending away from the observer in depth at approximately equal intervals. The lateral separation of the LEDs in each pair was reduced as the distance of the pair from the observer increased. The LEDs were positioned along a tabletop and $28.5 \mathrm{~cm}$ below the observer's eye level. With all the LEDs turned on in an otherwise completely darkened visual alley, the appearance of the display was similar to that of a simplified airport runway seen head-on at night. (The apparent depth within the configuration was somewhat increased by the physical reduction in the lateral separation of the LEDs as the pairs were positioned farther from the observer. Consequently, both a binocular disparity of approximately $25 \mathrm{~min}$ of arc and an exaggerated linear perspective were used to create the apparent depth between the front and the back of the display.)

The LEDs were all matched approximately in brightness at a level sufficient to maintain the surrounding darkness. (No reflections from other surfaces were ever visible under the conditions of adaptation used, because the floor and the walls of the alley were covered with dark cloth.) The LEDs could be turned on in the following combinations: the pair of nearest (green) LEDs, the pair of farthest (green) LEDs, both the near and far pairs, or the near and far pairs and all of the intermediate pairs of yellow-orange LEDs.

Displays were always observed from inside a booth adjacent to the alley but separated from it by a wall containing binocular viewing apertures (each $2 \mathrm{~cm}$ wide $x 1 \mathrm{~cm}$ high) with a center-tocenter separation of $6.5 \mathrm{~cm}$. These apertures were edged with opaque film to eliminate the possibility of seeing the openings themselves. A headrest and chin rest positioned the observer. A shutter occluded the display until the appropriate time. The observation booth also contained an intercom system through which the observer and the experimenter could talk to each other and through which white noise could be presented to the observer.

\section{Observers}

The observers for the experiment were 71 male and 25 female undergraduate students enrolled in introductory psychology $(\mathrm{N}=96)$. They were given credit for participating as one means of satisfying a research requirement for their course. All observers had near and far visual acuity of at least 20/30 in both eyes (corrected if necessary) and a stereo acuity of at least $145 \mathrm{sec}$ as measured on a Keystone Telebinocular and with a Keystone Multi-Stereo Test. All observers were naive with respect to the purpose of the experiment.

\section{Procedure}

The observers were divided randomly into four equal groups. One group was presented the nearest pair of points alone. Another group was presented the farthest pair of points alone. The third group was presented both the near and far pairs of points simultaneously. The fourth group was presented the near and far pairs of points and the four pairs of intermediate (yellow-orange) LEDs simultaneously.

Each observer was screened for visual acuity before being taken into the observation booth and given instructions about the experimental task. One minute of dark adaptation then preceded the presentation. During this delay, white noise was delivered to the observer's earphones, in order to mask extraneous sounds. Following the period of darkness, the experimenter turned on the appropriate display, raised the shutter covering the observer's viewing apertures, and turned off the white noise. The experimenter asked whether all lights appeared to be at the same or at different distances from the observer and determined how many lights were seen by the observer. The experiment continued only for those observers who indicated by their responses to these questions that they had achieved the necessary binocular fusion. (Only a few observers needed to be replaced for a problem at this point.) The experimenter then asked the observer to describe the perceived distance or distances to certain objects in the configuration. In no case was an observer asked to make a distance judgment to the intermediate yellow-orange LEDs. Each observer did, however, make a judgment of the apparent distance to one pair or to both pairs of green LEDs (depending upon the display being viewed). Judgments of distance were made as verbal reports "in feet or inches or in some combination of feet and inches." For those observers who were presented more than one pair of green LEDs, the order of reporting the apparent distances of the nearer or farther pairs was counterbalanced across observers. Each observer served in only one condition and received only one presentation of objects.

\section{RESULTS AND DISCUSSION}

The results of the experiment are shown in Table 1. The original reports of perceived distance $\left(D^{\prime}\right)$, expressed in feet and inches, have all been converted to centimeters. Since some of the distributions are skewed and/or contain a few extreme values (cf. Mershon \& Lembo, 1977, for a discussion of this problem in analyzing verbal reports), medians rather than arithmetic means best represent these data. Geometrical mean (GM) values, which are also less sensitive to skew, have been presented for additional comparison. The data shown in Table 1 for single presentations indicate the apparent distances of the near and far points when each pair was presented alone. It is clear from these results that no significant cues to egocentric distance existed in this situation. The near points and far points were not significantly different by a Mann-Whitney U test. In fact, the reported position of the near points slightly exceeded that of the far points when each pair was presented alone. Hence, we may use an average of these values 
Table 1

Medians, Semiinterquartile Ranges, and Geometrical Means of Perceived Distance $\left(D^{\prime}\right)$ for Single and Simultaneous Presentations of the Near and Far Binocular Points

\begin{tabular}{|c|c|c|c|c|c|c|c|}
\hline \multirow[b]{3}{*}{ Configuration } & & & & \multicolumn{4}{|c|}{ Simultaneous Presentations } \\
\hline & \multicolumn{3}{|c|}{ Single Presentation } & \multicolumn{2}{|c|}{ Near and Far Points } & \multicolumn{2}{|c|}{ All Points } \\
\hline & Near Point & Far Point & Average & Near Point & Far Point & Near Point & Far Point \\
\hline Median & 304.8 & 243.8 & 274.3 & 160.0 & 304.8 & 152.4 & 304.8 \\
\hline Semiinterquartile Range & 472.4 & 190.5 & & 110.5 & 228.6 & 41.9 & 213.4 \\
\hline Geometrical Mean & 432.0 & 384.1 & 408.0 & 189.9 & 420.9 & 164.6 & 388.7 \\
\hline
\end{tabular}

Note-All values have been converted to centimeters.

from the single presentations as an overall estimate for the egocentric reference distance in this study.

The primary experimental question was whether or not (when presented in a configuration) the near points, the far points, or both would be perceptually displaced from their position in isolation. These comparisons may be tested statistically in two ways. First, we may use the sign test to compare each distribution of values with the value of the ERD determined from the single-presentation data. This procedure indicates that the far points did not significantly change their apparent position relative to the ERD. When the near points were seen as part of a configuration, however, they were significantly shifted toward the observer relative to the ERD $(p=.032$, one-tailed, for the display consisting of both the near and the far points and $p=.001$, one-tailed, for the full display).

We may also test the displacement effect by utilizing the Mann-Whitney $U$ test to compare each distribution in the simultaneous presentations with the distribution of judgments for the same pair of points when presented in isolation. With this procedure, the near points presented with the full display were significantly closer than the near points alone $(U=187, \mathrm{z}$ corrected for ties $=$ $2.091, \mathrm{p}<.02$, one-tailed). The distribution of values for the near points presented with the far points (without the intermediate lights) approached but did not quite reach statistical significance $(U=209, z$ corrected for ties $=1.637, \mathrm{p}<.06$, one-tailed). The far points did not differ significantly for either simultaneous display. Although no statistical tests have been applied to the GM data, it is clear that their pattern supports this same interpretation.

It seems that the effect of presenting objects in a configuration that extends in depth is approximately the same regardless of whether intermediate objects are presented. The nearest objects in the configuration are perceptually displaced toward the observer from their position when seen in isolation. The amount of this displacement is also approximately the same. Although this result may seem somewhat surprising, it nevertheless confirms both the generality of the previous findings and the importance of the location of the farthest object in a configuration. When minimal cues to the egocentric distances of the individual parts of the display are avail- able, the farthest object(s) will tend to be seen at a fairly fixed distance (the ERD) and closer objects (to the extent that relative depth cues exist in the situation) will be seen arranged in depth accordingly. Thus the perceptual scale for a display of objects under reduced viewing conditions will be strongly determined by the positioning of the farthest object in the configuration.

With regard to the generality of this phenomenon, it must be noted that post hoc separations of the data by sex occasionally do suggest some variability not found in the main groups. Thus, in the present experiment, female subjects reported that both near and far points were significantly closer when seen together than when seen alone. Male subjects reported that the far points were perceptually farther when seen with the near points than when seen alone, whereas the near points were not significantly shifted in apparent distance from their position in isolation. The latter result was significant by Mann-Whitney $\mathrm{U}$ test $\mathrm{z}=1.71$, corrected for ties, $p<.05$, one-tailed) and, if replicable, would, of course, counter the general conclusion expressed above. However, the same comparisons within those male subjects presented the full display failed to reach significance, yielding instead the same pattern of results that was found for the main group. Furthermore, reanalyses of the raw data of the experiments of Mershon and Lembo (1977) and Mershon et al. (1976) also failed to provide evidence for any consistent sex-linked differences in the perceived distances of the objects in a configuration. Consequently, it still seems fair to characterize the most persistent feature of these studies as a relative perceptual stability of the farther portion of the display. (At this point, it might be wise to call for the use of groups explicitly balanced by sex in any future studies, so that this variable can be considered more systematically. There is not, however, any strong indication of the need for explicit research on sex differences within the context of the present problem.)

Still to be determined, of course, is the basis for the importance of the farthest object. One possible factor may be the observer's pattern of fixation in such a display. Observers in all the studies to date have used free fixation. It is conceivable that observers tend to fixate one end of the display or the other and that such fixation may itself partly determine the importance of 
a particular object to the perceptual results. An example of a similar effect was demonstrated by Gogel and Tietz (1977), who showed that the choice of fixation in a multicue display significantly affected the apparent distances of the objects presented. Our display was much more impoverished than theirs, however. Whether or not the perceived positioning of our binocular configuration of objects, relative to the ERD, is also determined by fixation remains to be investigated.

\section{REFERENCES}

GoGEL, W. C. The sensing of retinal size. Vision Research, 1969, 9, 1079-1094.
Gogel, W. C. Scalar perceptions with binocular cues of distance. American Journal of Psychology, 1972, 85, 477-497.

Gogel, W. C., \& TIETz, J. D. Eye fixation and attention as modifiers of perceived distance. Perceptual and Motor Skills, 1977, 45, 343-362.

Mershon, D. H., \& Lembo, V. L. Scalar perceptions of distance in simple binocular configurations. American Journal of Psychology, 1977, 90, 17-28.

Mershon, D. H., Voncannon, M. G., \& Windes, W. R. Scalar perceptions of distance for a monocularly determined depth interval. Bulletin of the Psychonomic Society, 1976, 8, 341-342. 